Received 12 May 2010 | Accepted 15 Nov 2010 | Published 11 Jan $2011 \quad$ DOl: 10.1038/ncomms1136

\title{
Sex-specific timing of meiotic initiation is regulated by Cyp26b1 independent of retinoic acid signalling
}

Sandeep Kumar', Christina Chatzi', Thomas Brade', Thomas J. Cunningham 1 , Xianling Zhao \& Gregg Duester ${ }^{1}$

Sex-specific initiation of meiosis in the fetal ovary has been suggested to require retinoic acid (RA) for induction of Stra8, with expression of the RA-degrading enzyme Cyp26b1 in fetal testis delaying meiosis until postnatal development. In this study, we investigate Raldh2-/- mice lacking RA synthesis and signalling in mesonephros and adjacent gonad and reveal that Stra8 expression in the fetal ovary does not require RA signalling. In contrast to previous observations, we find that Stra 8 is expressed in the absence of physiologically detectable levels of RA. Ketoconazole

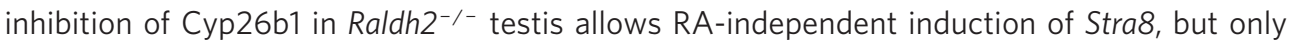
when the mesonephros remains attached, pointing to a non-RA signal from the mesonephros that induces Stra8 in the adjacent gonad. These findings demonstrate that Cyp26b1 prevents the onset of meiosis by metabolizing a substrate other than RA that controls Stra8 expression, thus changing the paradigm for how studies on Cyp26 function are conducted.

\footnotetext{
' Sanford-Burnham Medical Research Institute, Development and Aging Program, 10901 North Torrey Pines Road, La Jolla, California 92037, USA Correspondence and requests for materials should be addressed to G.D. (email: duester@sanfordburnham.org).
} 
$\mathrm{D}$ uring generation of haploid gametes, entry into meiosis exhibits a distinct sex-specific difference in timing, with female gametes being generated during late fetal development and male gametes being generated postnatally at puberty ${ }^{1}$. Entry into meiosis was proposed to be an intrinsic property of fetal germ cells, unless prevented by a meiosis-inhibiting factor produced in the fetal testis but not fetal ovary ${ }^{2}$. Recent findings demonstrated that supraphysiological levels of retinoic acid (RA) can stimulate premature meiosis in fetal testis ${ }^{3,4}$. Also, investigation of Cyp26b1null mutant mice demonstrated that the P450 enzyme Cyp26b1, normally expressed in Sertoli cells of fetal testis but not ovary, is required for delayed entry into meiosis in male mice ${ }^{4-6}$. As Cyp26b1 is known to degrade $\mathrm{RA}^{7}$, this suggested a role for RA as a sexspecific inducer of meiosis. During embryonic development, RA functions mainly in a paracrine manner, with RA synthesis being controlled mostly by tissue-specific expression of retinaldehyde dehydrogenase-2 (Raldh2), which generates RA that travels to RA target cells where it directly regulates genes via ubiquitous nuclear RA receptors (RARs) bound to RA response elements (RAREs) ${ }^{8}$. Expression of Raldh2 (Aldh1a2) in the mesonephros was reported to serve as a paracrine source of RA for the adjacent gonad, which does not express Raldh2 (ref. 4). Stra8 is a premeiotic gene required for meiotic initiation that is normally expressed only in ovaries during embryogenesis ${ }^{9}$. Stra 8 can be prematurely induced in testis by treatment with high levels of RA, whereas treatment of ovaries with RALDH2 inhibitors or RAR antagonists results in downregulation of Stra8 expression ${ }^{3,4}$. These findings generated a new model for sex-specific timing of meiosis entry, in which RA generated in the mesonephros serves as an extrinsic inducer of Stra8 in the adjacent gonad unless degraded by Cyp26b1 (ref. 10).

In order to ascertain whether endogenous RA normally has a role during induction of meiosis, we investigated Raldh $2^{-1-}$ mouse embryos lacking RA synthesis. In this study, we show that RA signalling is not required for induction of Stra8 expression in the fetal ovary. We also demonstrate that the critical role Cyp26b1 has in preventing onset of meiosis in the fetal testis does not involve degradation of RA, suggesting an RA-independent function of Cyp26b1 in control of meiotic initiation.

\section{Results}

Stra8 is unaffected by loss of RA synthesis in the fetal ovary. Loss of endogenous RA synthesis in Raldh $2^{-1-}$ mouse embryos results in the cessation of growth after E8.5 and death soon after ${ }^{11,12}$. Low-dose maternal dietary RA supplementation of Raldh2 $2^{-1-}$ embryos carried out for a limited time from E6.75 to E9.25 provides less RA than is normally produced by RALDH2 (ref. 13); however, this treatment rescues early lethality and allows the study of RA function in tissues of older embryos that normally exhibit RA activity but now lack such activity ${ }^{14,15}$. As RA administered to embryos is completely cleared within $12-24 \mathrm{~h}^{11}$, tissues that require RALDH2 for their endogenous RA will lack RA activity from at least E10.25 onwards using a treatment regimen ending at E9.25. In the studies reported here, RA treatment of Raldh2 $2^{-l-}$ embryos ended 3 days before initiation of Stra8 expression in the ovary, which occurs at E12.5 (ref. 16). We observed that Raldh $2^{-/-}$embryos rescued in this manner did not exhibit a loss or reduction of Stra8 expression in E13.5 fetal ovaries (Fig. 1a-d). Although Raldh2 is primarily responsible for RA synthesis throughout the mesonephros, Raldh3 (Aldh1a3) is expressed at E10.5 in a limited region of the mesonephric duct that gives rise to the ureteric buds of the kidney ${ }^{14}$. Therefore, we

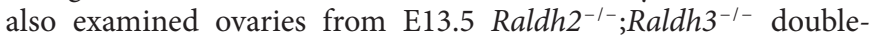
mutant embryos (rescued as described above), and found that Stra 8 expression still remained robust and was comparable with wild-type or rescued Raldh2 $2^{-/-}$single-mutant ovaries (Fig. 1e,f). Sections of single- and double-mutant E13.5 ovaries revealed no reduction in Stra8 expression relative to wild-type (Fig. 1b,d,f).
We also examined Stra8 expression at additional time points, as it has been reported that expression normally initiates at E12.5 (ref. 16). Stra8 expression was not observed in either wild-type or Raldh2 $2^{-1-}$ mutant ovaries at E12.0, verifying that RA-rescued mutants treated with a low dose of RA until E9.25 do not exhibit premature induction of Stra8 (Fig. 1g,h); our previous studies using the same regimen for generating RA-rescued Raldh 2 mutants have shown that following low-dose RA treatment ending at E9.25, the mutants are clearly RA deficient by at least E12.5, as RA activity normally present in the interdigital mesenchyme is completely missing and forelimbs are stunted ${ }^{15}$. At E12.5, weak Stra8 expression was observed (Fig. 1i,j) and at E14.5 much stronger Stra 8 expression was observed (Fig. 1k,l), thus demonstrating that Raldh2 $2^{-/-}$mutant ovaries exhibit normal induction and maintenance of Stra8 expression. Thus, in contrast to previously reported RA inhibitor and antagonist studies ${ }^{3,4}$, our genetic experiments reveal that the enzymes synthesizing RA in the vicinity of the ovary are not required for Stra8 expression in the ovary.

Previous studies have demonstrated that E13.5 testes lack Stra8 expression due to expression of Cyp26b1 (refs 3, 4). We observed no Stra8 expression in E13.5 wild-type testis and this was unchan-

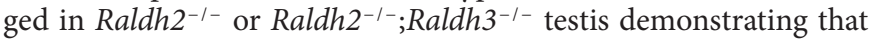
RA is not required to repress male Stra 8 expression at this stage (Fig. 1m-o).

RA is not required for induction of meiosis. In order to determine whether RA signalling is required for the initiation of meiosis, we examined E13.5 wild-type and Raldh2 ${ }^{-1-}$ ovaries for expression of $S c p 3$ encoding a synaptonemal complex protein expressed during meiotic prophase ${ }^{17}$. Whole-mount in situ hybridization demonstrated similar expression of Scp3 in wildtype and Raldh2 $2^{-1-}$ ovaries (Fig. 2a,b). To confirm our observation that RA is not required for meiotic initiation in embryonic ovary, we examined E13.5 wild-type and Raldh $2^{-1-}$ ovaries for the presence of phosphorylated $\mathrm{H} 2 \mathrm{AX}(\gamma-\mathrm{H} 2 \mathrm{AX})$, a histone $\mathrm{H} 2 \mathrm{~A}$ isoform, which is phosphorylated in response to DNA double-strand breaks in the cell during meiotic recombination ${ }^{9,18}$. Immunostaining for $\gamma$-H2AX demonstrated a similar distribution of DNA doublestrand breaks throughout both wild-type and Raldh2-/- ovaries, indicating that germ cells in Raldh $2^{-1-}$ ovaries are engaged in meiotic recombination (Fig. 2c,d); wild-type testis was negative for $\gamma$-H2AX immunostaining (Fig. 2e). In addition, analysis of DAPI-stained nuclei demonstrated that E14.5 wild-type and Raldh2 $2^{-1-}$ ovaries both exhibit germ cells with condensed nuclei characteristic of meiotic prophase (Fig. 2f,g). Together, these findings provide evidence that RA is not required for induction of meiosis during ovary development.

Raldh mutants lack RA activity in mesonephros/gonad. Examination of E12.0 and E13.5 wild-type and Raldh2 ${ }^{-1-}$ embryos carrying the RARE-lacZ RA-reporter transgene ${ }^{19}$ revealed that RA activity typically observed throughout the wild-type mesonephros was totally eliminated in the mutant, while no RA activity was detected in either wild-type or mutant ovaries (Fig. 3a-d). Thus, the RA rescue treatment for Raldh2-/- embryos does not result in RA activity in the mesonephros/ovary at E12.0, which is prior to the time when Stra8 expression normally initiates. Kidneys from E13.5 Raldh2 $2^{-1-}$ embryos were smaller than wild type and retained some RA activity (presumably due to Raldh3 expression in the ureteric buds), providing further verification that mutants were carrying the $R A R E$ lac $Z$ transgene (Fig. 3c,d). As an independent test of RA activity, we performed tissue explant RA bioassays ${ }^{20}$, in which ovary or mesonephros tissues were dissected from E13.5 wild-type, Raldh2 $2^{-/-}$ and Raldh $2^{-1-} ;$ Raldh $^{-1-}$ embryos and cultured on a monolayer of F9 RA-reporter cells (incorporating a RARE-lacZ transgene). The results of this experiment were consistent with our in vivo 

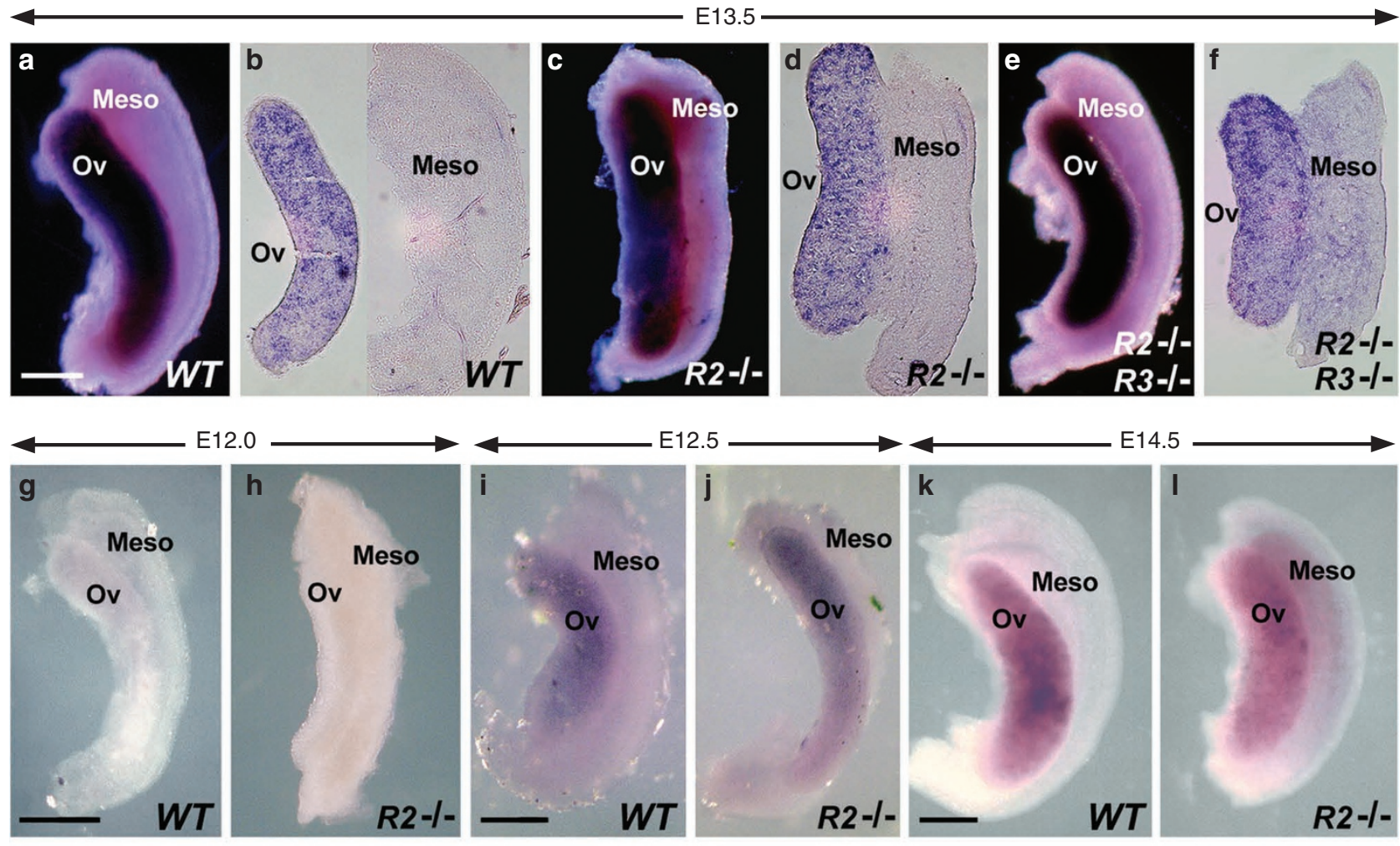

E12.0

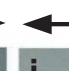

E12.5

E14.5
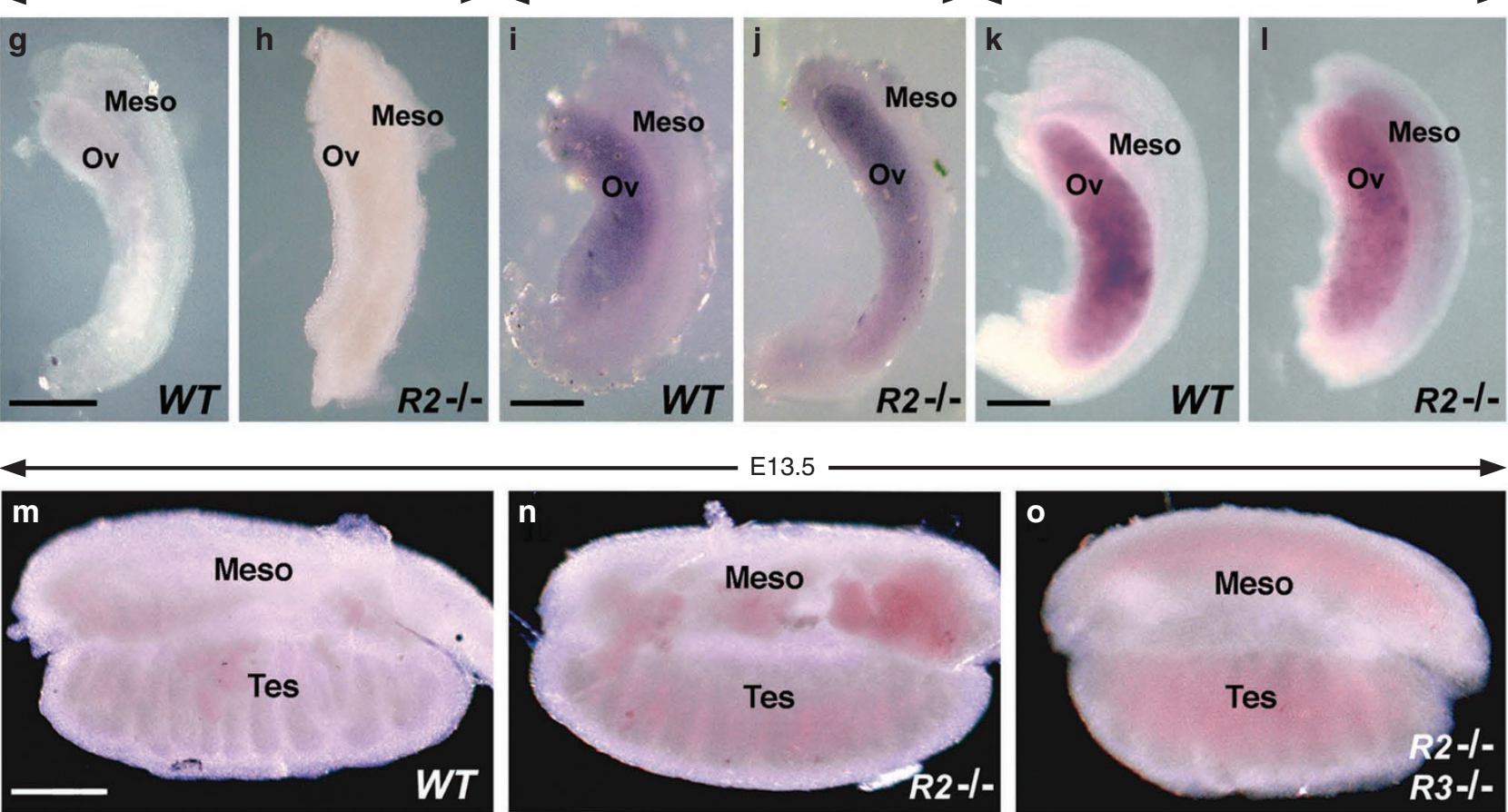

Figure 1 | Genetic loss of RA synthesis does not affect Stra8 expression in fetal ovary. Stra8 mRNA was detected in gonads by whole-mount in situ

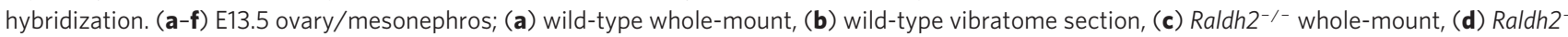

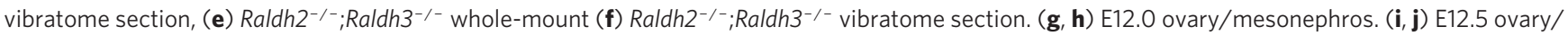
mesonephros. (k, I) E14.5 ovary/mesonephros. (m-o) E13.5 testis/mesonephros. Meso, mesonephros; ov, ovary; tes, testis. $n=3$ for all wild-type (WT)

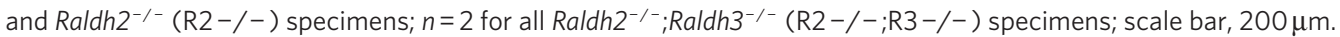

RARE-lacZ expression data, confirming that only wild-type mesonephros possesses RA activity, whereas single/double-mutant mesonephros and either wild-type or single/double-mutant ovaries completely lack RA activity (Fig. 3e). Together with our Stra 8 expression data, these findings show that the mesonephros

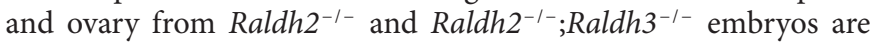
devoid of any RA signalling, thus demonstrating that Stra8 expression in the ovary does not require RA signalling.

RARE-lacZ as a sensitive indicator of RA in ovary. Our findings above suggest that Stra8 does not require RA for expression in the ovary, as this organ normally has no RA activity detectable by $R A R E$-lacZ. We performed studies to determine if the RARE-lacZ transgene is able to detect physiological levels of RA in the ovary by culturing wild-type ovaries in the absence or presence of physiological levels of RA. Previous high-performance liquid chromatography measurements have shown that physiological RA concentrations in various regions of E13.5 mouse embryos range from 10 to $40 \mathrm{nM}$ (except $100 \mathrm{nM}$ in spinal cord), with the combined visceral organs having $\sim 30 \mathrm{nM}^{21}$; also, high-performance liquid chromatography studies on whole E10.5 embryos revealed a concentration $\sim 25 \mathrm{nM}^{13}$. Thus, ovaries were treated with physiological amounts of RA from 25 to $100 \mathrm{nM}$. Ovaries cultured without RA resulted in no expression of RARE-lacZ in the ovary, but treatment with 25 or $100 \mathrm{nM}$ RA was able to induce RARE-lacZ (Fig. $4 \mathrm{a}-\mathrm{c}$ ). Similar treatment of wild-type testes did not induce RARE-lac $Z$ at either RA concentration presumably due to expression of Cyp26b1 (Fig. 4d-f). As $R A R E-l a c Z$ was able to be induced in ovary by treatment with as little as $25 \mathrm{nM}$ RA, these findings demonstrate that RARE-lac $Z$ is indeed able to detect physiological levels of RA in the ovary, and that the absence of RARE-lacZ expression in ovary indicates that this organ does not contain enough RA to stimulate RA signalling in vivo. These findings further suggest that RA synthesized in the mesonephros does not travel to the ovary in an amount sufficient to stimulate RA signalling in this tissue.

RA-independent function for Cyp26b1 in Stra8 induction. Expression of Stra8 requires a germ cell intrinsic factor encoded by 


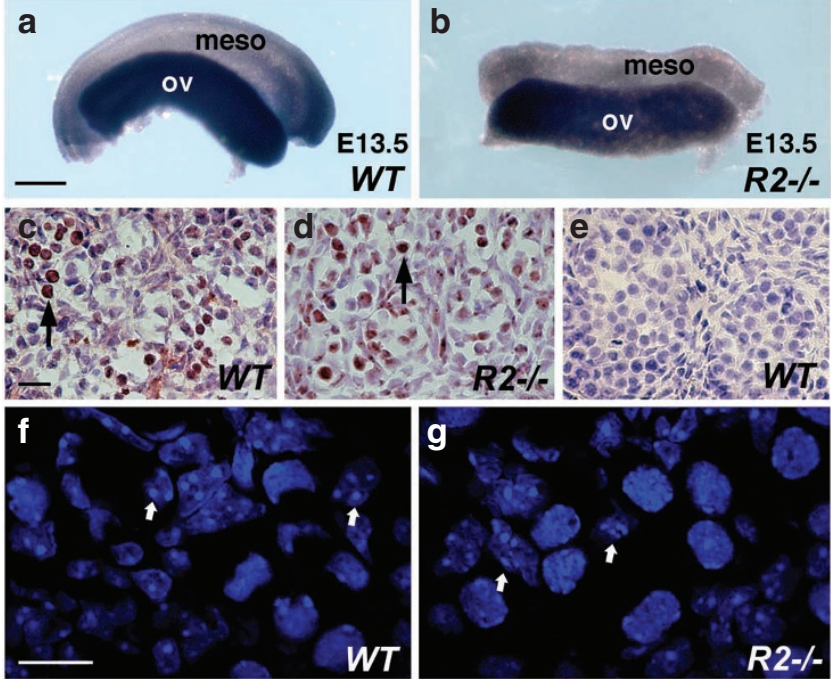

Figure $\mathbf{2}$ | Germ cells in Raldh2 mutant ovary undergo meiosis. (a, b)

Expression of Scp3 in E13.5 wild-type and Raldh2-/- ovaries examined by whole-mount in situ hybridization; scale bar, $200 \mu \mathrm{m}$. (c-e) Immunostaining for $\boldsymbol{\gamma}-\mathrm{H} 2 \mathrm{AX}$ in E13.5 ovary (c, d) and testis (e); arrows indicate female germ cells positive for $\gamma-\mathrm{H} 2 \mathrm{AX}$ detection; scale bar, $10 \mu \mathrm{m}$. (f, $\mathbf{g})$. Confocal images of nuclei stained with DAPI (4',6-diamidino-2-phenylindole) in E14.5 wildtype and Raldh2-/- ovaries; arrows indicate representative germs cells in meiotic prophase; scale bar, $10 \mu \mathrm{m}$. Meso, mesonephros; ov, ovary. $n=3$ for each specimen.

Dazl and an extrinsic factor suggested to be RA ${ }^{22}$. Fetal testis normally lacks Stra 8 expression due to the presence of the P450 enzyme Cyp26b1, and treatment of cultured testis/mesonephros with the P450 inhibitor ketoconazole is conducive for Stra8 expression ${ }^{3,4}$. As Cyp26b1 has been associated with RA degradation, it was postulated that Cyp26b1 inhibitors permitted diffusion and accumulation of RA from the mesonephros to the testis, to prematurely induce Stra8. In order to explore this possibility, we first observed that the E13.5 Raldh2 -l- $^{-}$testis/mesonephros complex completely lacked RA signalling according to RARE-lacZ analysis (Fig. 5a). This finding supports the view that the mesonephros from either sex predominantly uses Raldh2 for RA synthesis. Treatment of cultured E13.5 intact testis/mesonephros complexes with ketoconazole induced Stra 8 in wild-type testis as expected, but we also observed equivalent Stra 8 induction in Raldh2 $2^{-1-}$ testis, which lacked a source of RA in the adjacent mesonephros (Fig. 5b). Furthermore, when testes were carefully separated from mesonephros and cultured alone in the presence of ketoconazole, Stra 8 was not induced in either wild-type or Raldh2 $2^{-/-}$backgrounds (Fig. 5b). Previous studies on ketoconazole-treated testis ${ }^{3}$ or Cyp26b1-1- testis $^{4}$ did not examine whether testis RA activity was increased as would have been expected if RA is needed to induce Stra8. Interestingly, we observed that RARElacZ RA activity was not detected in E13.5 wild-type testis cultured in the presence of ketoconazole under the same conditions described above that induce Stra8 (Fig. 5c). Thus, RA degradation by Cyp26b1 is not the mechanism preventing RA activity in embryonic testis; perhaps RA is not efficiently transported into the testis from the mesonephros similar to what we observed for ovary (Fig. 4). Together, these findings demonstrate that RA is not required for Stra8 induction in testis, but imply that some other extrinsic signal from the mesonephros is required and Cyp26b1 blocks the action of that signal.

Inefficient recruitment of RARs to Stra8 promoter in ovary. Stra8 was first identified as an RA target gene in experiments involving supraphysiological doses of RA administered to mouse embryonal carcinoma cells ${ }^{23}$. In addition, Stra8 expression in the testis can be induced by RA treatment, but this requires high levels of exogenous RA that are non-physiological ${ }^{3,4}$. A 400 bp promoter region upstream of mouse Stra8, containing a DNA sequence at -210 bp resembling a DR2 RARE (direct repeat with 2 bp spacer), has been reported to be sufficient to direct spermatogonia-specific expression of Stra8 in postnatal transgenic mice, although it was not demonstrated that the putative RARE is required for Stra 8 expression ${ }^{24}$. Here, we performed chromatin immunoprecipitation (ChIP) studies to investigate the ability of this putative Stra8 DR2 RARE to recruit any of the three RARs compared with the RARb RARE used to construct $R A R E-l a c Z$, that is, a DR5 RARE (direct repeat with 5 bp spacer) located at $-53 \mathrm{bp}$ in the mouse $R A R b$ promoter, which is known to be induced by RA in vivo to increase RARs as part of the RA signalling response ${ }^{25}$. ChIP performed on E13.5 fetal ovary/mesonephros tissue demonstrated that the putative Stra8 RARE exhibits no detectable binding to RAR $\alpha$, and a relatively low level of binding to RAR $\beta$ and RAR $\gamma$ (Fig. 6a,b). In comparison, the RARb RARE exhibited strong binding to all three receptors RAR $\alpha, \operatorname{RAR} \beta$ and RAR $\gamma$ in the same ovary sample (Fig. 6c). A quantitative comparison of the relative binding efficiencies shows that the Stra8 RARE does not bind RAR $\alpha$ above background levels and it binds RAR $\beta$ and RAR $\gamma$ about 5 times less efficiently than the RARb RARE (Fig. 6d). If a RARE is needed for Stra8 expression, it should be present in the promoter proximal $400 \mathrm{bp}$ region, previously shown to be sufficient for germ cell expression in vivo ${ }^{24}$. However, an in silico approach was used to analyse $-10 \mathrm{~kb}$ upstream and $+10 \mathrm{~kb}$ downstream of the mouse Stra8 gene for the presence of any other sequences resembling a RARE. We identified a putative DR1 RARE (direct repeat with 1 bp spacer) located at $-742 \mathrm{bp}$ upstream of the Stra 8 transcription start site, but we detected no binding of any RARs to this sequence using ChIP analysis on E13.5 ovary/mesonephros. These findings demonstrate that the Stra8 regulatory region is less effective in binding RARs than the RARb RARE present in the RARE-lac $Z$ transgene used here to detect RA activity. This observation provides further evidence that RARE-lacZ is more sensitive to RA induction than Stra8, thus supporting our hypothesis that Stra8 expression in fetal ovary does not require RA.

\section{Discussion}

Our studies provide evidence that gonadal Stra8 expression and induction of meiosis does not require endogenous RA. We dem-

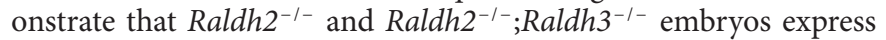
Stra8 in embryonic ovary, despite no RA activity detected in mesonephros/ovary using either embryos carrying the RARE-lacZ transgene or a tissue explant assay utilizing an RA reporter cell line harbouring $R A R E$-lacZ. Although $R A R E$-lacZ is expressed in wild-type mesonephros, it is not expressed in wild-type ovary raising the concern that this transgene may not function in ovary for some reason other than a lack of RA. However, we demonstrate that $R A R E-l a c Z$ is able to detect physiological levels of RA in the ovary by showing that RARE-lacZ can be induced in ovary by treatment with as little as $25 \mathrm{nM}$ RA, thus strengthening our argument that RA signalling does not normally occur in the ovary and thus cannot be required for Stra 8 induction. RARE-lacZ contains multiple copies of the efficient RARE found upstream of the $R A R b$ gene, which we demonstrate here by ChIP to be much more efficient than the putative RARE upstream of Stra8. Thus, if a very low level of RA remains

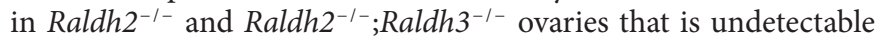
by RARE-lacZ, our findings suggest that Stra 8 would be unable to respond to such low levels.

Our observation that E13.5 wild-type ovary does not exhibit detectable RA activity using the F9 RA-reporter cell assay contradicts a previous report suggesting that the ovary contains nearly as much RA as mesonephros using this assay ${ }^{4}$; in those studies we suggest that the ovary may not have been cleanly separated from the 


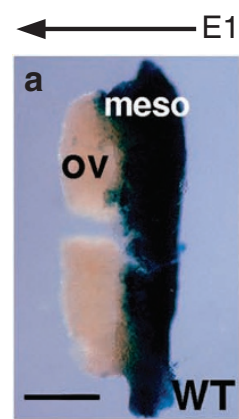

E12.0

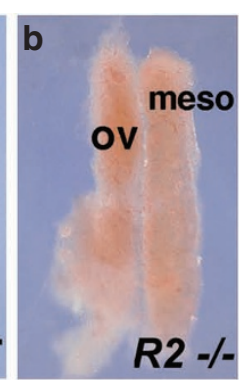

e
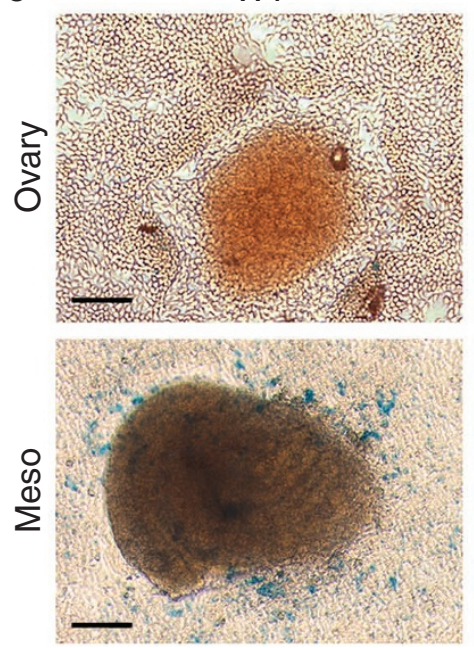

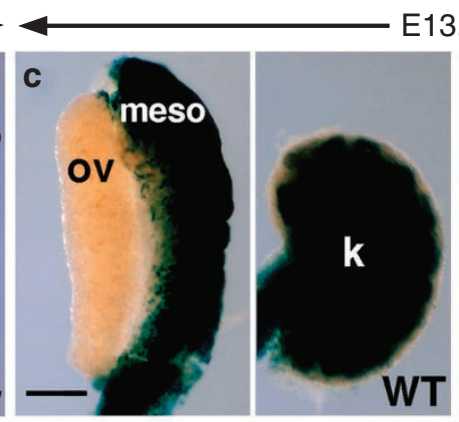

Raldh2-1-
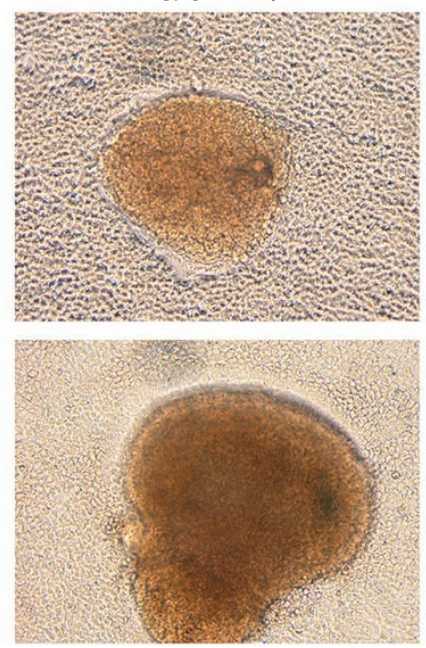

13.5

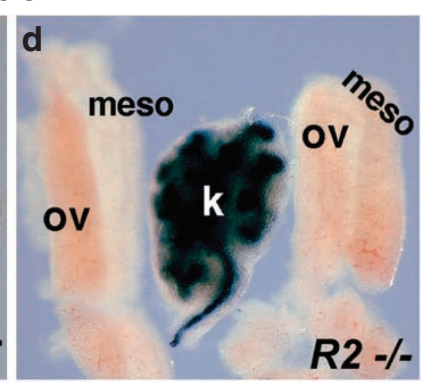

Raldh2;Raldh3-/-
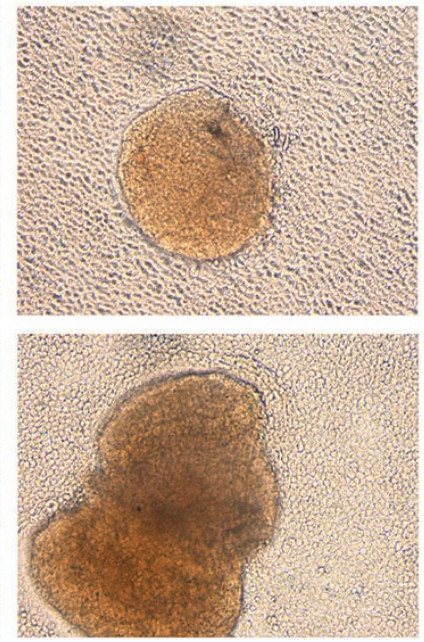

Figure 3 | Mesonephros and gonads of Raldh mutants exhibit no RA signalling activity. Detection of RA activity in embryonic ovary/mesonephros. (a, b) RARE-lacZ expression in E12.0 mesonephros/ovary of wild-type (WT) and Raldh2-/- (R2-/-) embryos; $n=2 ;$ scale bar, $200 \mu \mathrm{m}$. (c, d) RARE-lacZ expression in E13.5 mesonephros/ovary; staining in kidney from the same embryo verifies that the embryo carries RARE-lacZ; $n=3 ; \mathrm{scale} b a r, 200 \mu \mathrm{m}$.

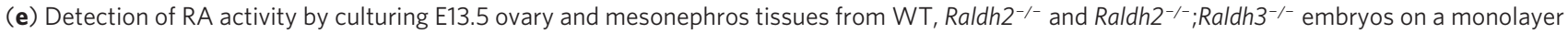
of F9 RA-reporter cells followed by staining for $\beta$-galactosidase activity; this experiment was performed using multiple tissue fragments in duplicate experiments with similar results as shown here; scale bar, $50 \mu \mathrm{m}$. k, kidney; meso, mesonephros; ov, ovary.

mesonephros as was done in our studies where we discarded ovary tissue directly adjacent to the mesonephros. Also, previous studies with the RARE-lacZ transgene reported that a very small amount of RA activity is detected at the anterior tip of the wild-type ovary at E13.5, presumably by diffusion of RA synthesized in the mesonephros by Raldh2, as the ovary itself does not express RA-generating enzymes ${ }^{4}$. However, no such RARE-lacZ activity was observed in our studies of E13.5 wild-type ovaries, suggesting that RA cannot efficiently travel from the mesonephros to the ovary. Even if small levels of RA can reach the anterior ovary by E13.5, this limited region of RA signalling does not correlate with widespread expression of Stra8 throughout the anterior-posterior length of the ovary at E13.5. Also, as loss of RA synthesis in Raldh2 ${ }^{-1-}$ embryos completely eliminates the large amount of RA activity present in the mesonephros, any diffusion of RA that may normally occur from mesonephros to the

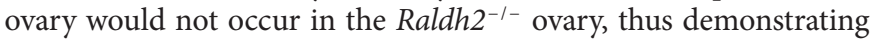
that RA is unnecessary for Stra8 expression observed throughout the anterior-posterior extent of mutant ovaries.

Endogenous RA is estimated to be in the $10-40 \mathrm{nM}$ range in most RA target tissues of mouse embryos ${ }^{13,21}$ and $11 \mathrm{nM}$ in adult testis where sperm are actively undergoing meiosis ${ }^{26}$. In previous studies, Stra8 was found to be induced in cultured fetal testis following treatment with supraphysiological doses of RA in the range of 25- to 50-fold higher than endogenous levels, i.e., $0.7 \mu \mathrm{M} \mathrm{RA} \mathrm{A}^{3,22}$ or $1.0 \mu \mathrm{M} \mathrm{RA}{ }^{4}$. We suggest that high levels of RA may induce Stra8 through the weak RARE located upstream of its promoter, but our ChIP studies suggest that Stra8 is not normally a target of RA as its promoter binds RARs very weakly and may thus be unable to respond to endogenous levels of RA. We also suggest that findings from studies utilizing RAR antagonists ${ }^{3,4}$ may not reflect the physiological role of endogenous RA, as such compounds have been shown to exert non-specific effects on other nuclear receptors, including one documented case, in which an RAR $\alpha$ antagonist was shown to also induce PPAR $\gamma^{27}$. In addition, compounds such as citral or disulfiram, which are often used to inhibit RALDHmediated metabolism of retinaldehyde to $\mathrm{RA}^{4}$, will inhibit metabolism of other aldehyde compounds by 15 additional members of the aldehyde dehydrogenase family with functions other than RA

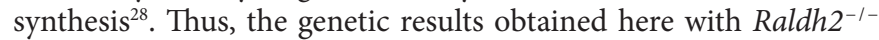
and Raldh $2^{-1-} ;$ Raldh $^{-1-}$ embryos, demonstrating that RA is not required for meiotic initiation, are less prone to artifacts and misinterpretation compared with these other approaches.

Although the genetic loss-of-function studies for Cyp26b1 clearly show that expression of this P450 enzyme in Sertoli cells is required to delay meiosis in the testis ${ }^{4-6}$, our findings reveal that one should not assume that Cyp26b1 functions only in the degradation of RA. Instead, we suggest that Cyp26b1 functions either by degrading an as yet unknown inducer of Stra 8 derived from the mesonephros or by synthesizing an inhibitor of Stra8 in the testis. Although the former hypothesis is supported by our testis/mesonephros explant studies suggesting that a factor other than RA travels from the mesonephros to the testis to induce Stra8, the latter hypothesis finds support from older studies suggesting the existence of a meiosisinhibiting factor produced in the fetal testis ${ }^{2}$. The latter hypothesis 
is also supported by recent studies suggesting that Sertoli cells may secrete a meiosis-preventing substance ${ }^{29}$, and that high levels of RA $(1-10 \mu \mathrm{M})$ may inhibit meiosis-preventing substance action ${ }^{30}$. Accordingly, high levels of RA may act as a competitive substrate inhibitor for some other activity of Cyp26b1, providing a nongenomic mechanism to explain the ability of supraphysiological RA to induce Stra8. In addition, recent findings have shown that
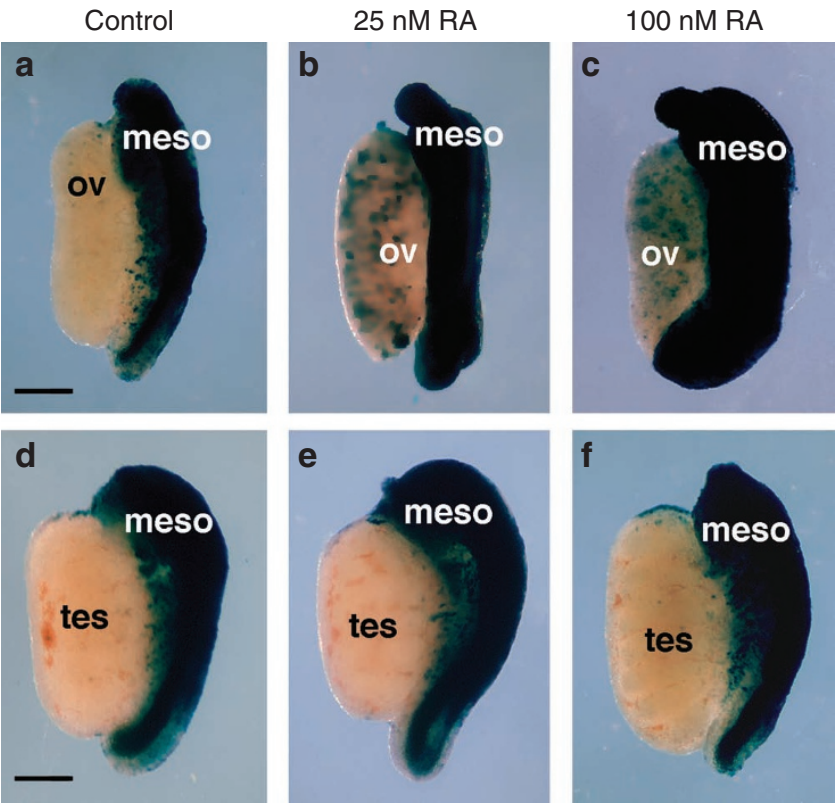

Figure 4 | Validation of RARE-lacZ transgene sensitivity to detect gonadal RA activity. RA activity detected by RARE-lacZ expression in wild-type E13.5 gonads cultured in the absence (control) or presence of a physiological dose of RA (25 or $100 \mathrm{nM}$ ). (a-c) Ovary/mesonephros. (d-f) Testis/mesonephros. Meso, mesonephros; ov, ovary; tes, testis. $n=4$ for each condition; scale bar, $200 \mu \mathrm{m}$. naive ovary cultured separately from mesonephros in the absence of RA spontaneously induces Stra8 and initiates meiosis ${ }^{31}$, and $\mathrm{Fgf9}^{-/-}$ fetal testis expresses Stra 8 and undergoes meiosis despite retaining Cyp26b1 expression ${ }^{32}$, further suggesting that RA is not required to induce Stra8. Our observation of Stra8 expression in Raldh2 gonads lacking RA synthesis thus provides important information needed to guide future studies toward understanding the role of Cyp26b1 in sex-specific timing of meiotic initiation.

\section{Methods}

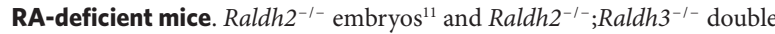
homozygous embryos ${ }^{14,33}$ were previously described. Single and double mutants were identified by PCR genotyping of yolk sac DNA. Rescue of embryos by maternal dietary RA supplementation was performed as previously described ${ }^{15}$ with low RA doses demonstrated to provide embryos an amount of RA in the normal physiological range ${ }^{13}$. The final RA concentration added to mouse chow was $0.1 \mathrm{mgg}^{-1}$ food, and treatment was from E6.75 to E9.25; all embryos analysed (wild-type and mutant) were from parents housed together that received the same brief RA treatment. At E9.25, pregnant mice were returned to standard mouse chow until the point of analysis at E12.0-E14.5. Rescued Raldh2 $2^{-/-}$embryos were easily identified by stunted forelimbs and exhibited defects in hindlimb interdigital development, as previously described ${ }^{15}$. All mouse studies conformed to the regulatory standards adopted by the Animal Research Committee at the Sanford-Burnham Medical Research Institute.

\begin{abstract}
Whole-mount in situ hybridization and immunohistochemistry. Whole-mount in situ hybridization was performed on gonads dissected from wild-type and mutant embryos at E12.0-E14.5. Gonads were fixed in $4 \%$ paraformaldehyde in phosphatebuffered saline (PBS) overnight, followed by in situ hybridization with Stra8 and $S c p 3$ digoxigenin-labeled antisense riboprobes prepared as described previously ${ }^{34}$. Stained whole-mount gonads were post-fixed for $1 \mathrm{~h}$ with $4 \%$ paraformaldehyde in PBS, then embedded in $3 \%$ agarose and sectioned at $30 \mu \mathrm{m}$ with a vibratome. For immunohistochemistry, dissected gonads were fixed in $4 \%$ paraformaldehyde, cryopreserved in $30 \%$ sucrose overnight at $4^{\circ} \mathrm{C}$ and then embedded in Tissue-Tek OCT compound (Sakura Finetek), oriented, and stored at $-80^{\circ} \mathrm{C}$. Cryosections were cut at a thickness of $12 \mu \mathrm{m}$, and immunostaining was performed on cryosections using 1:100 $\left(0.01 \mathrm{mg} \mathrm{ml}^{-1}\right)$ anti- $\gamma \mathrm{H} 2 \mathrm{AX}$ (Upstate Biotechnology) and visualized with 3',3'-diaminobenzidine Substrate Kit (Vector Laboratories). Sections were examined by bright field microscopy using a Nikon LABOPHOT-2 microscope.
\end{abstract}

Detection of RA activity. The RARE-lacZ RA-reporter transgene, which places lacZ (encoding $\beta$-galactosidase) under the control of a RARE, was used for in situ a
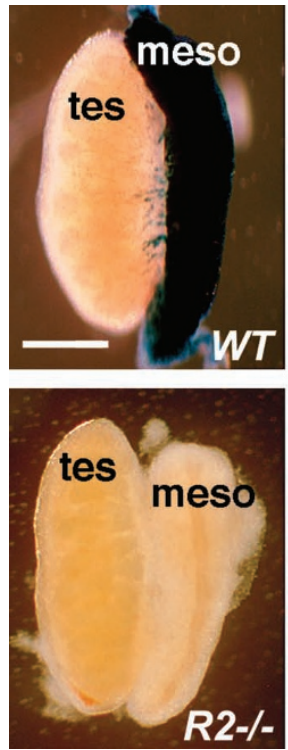

b
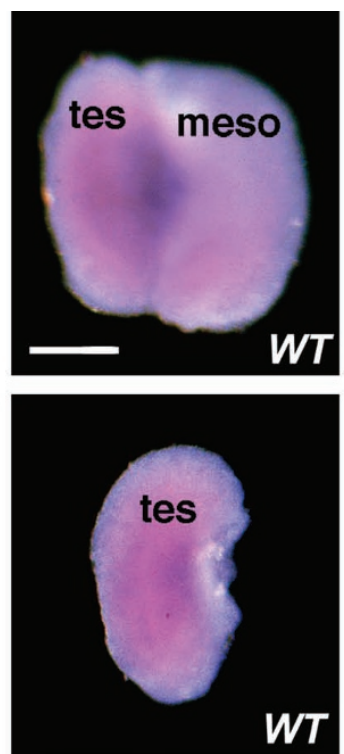

$0.7 \mu \mathrm{M}$ Keto
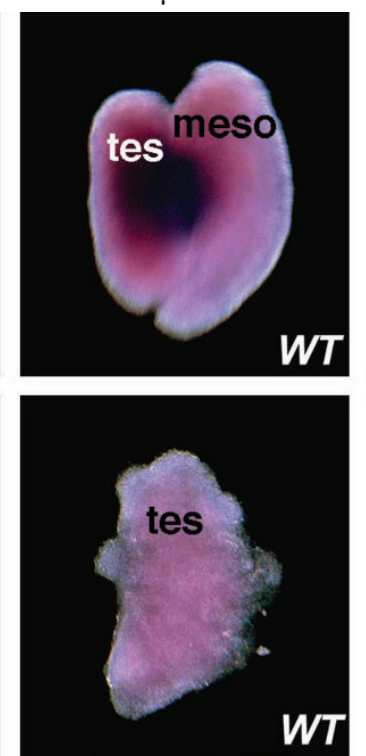

$0.7 \mu \mathrm{M}$ Keto
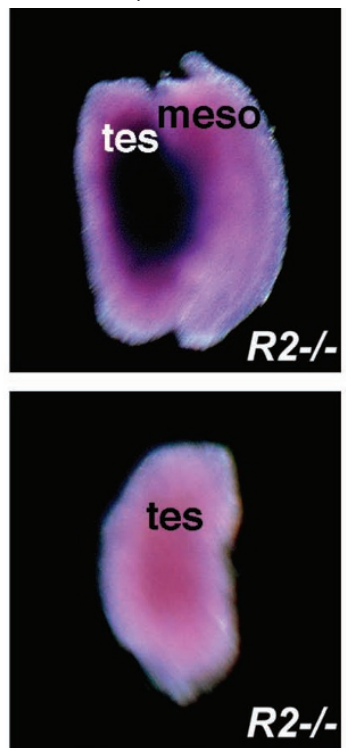

C
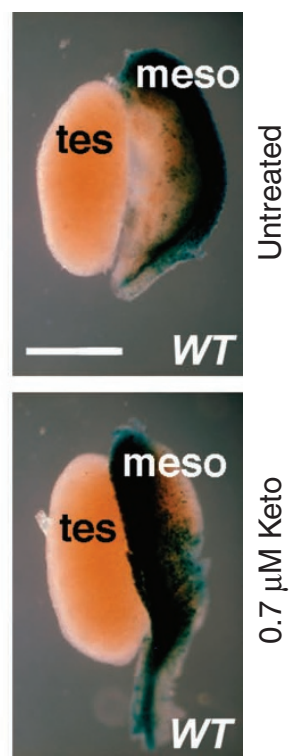

Figure 5 | RA-independent induction of Stra8 in Raldh2 mutant testis. (a) Detection of RA activity by analysis of RARE-lacZ expression in testis/mesonephros of E13.5 wild-type (WT) and Raldh2-/- (R2-/-) embryos; $n=3$. (b) Intact testis/mesonephros complexes or separated testis from E13.5 wild-type (WT)

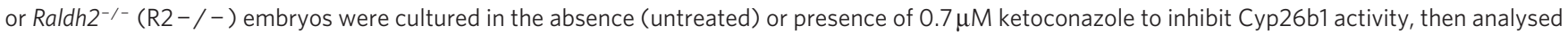
for Stra8 mRNA; similar results were obtained in duplicate experiments. (c) RARE-lacZ staining of wild-type E13.5 testis/mesonephros cultured in the absence (untreated) or presence of $0.7 \mu \mathrm{M}$ ketoconazole; $n=2$. Meso, mesonephros; tes, testis; scale bar, $200 \mu \mathrm{m}$. 

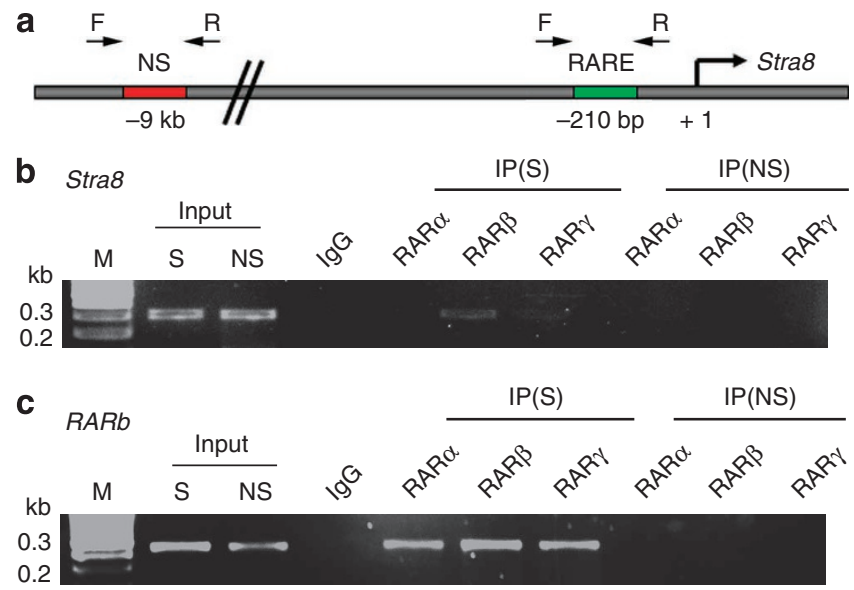

d

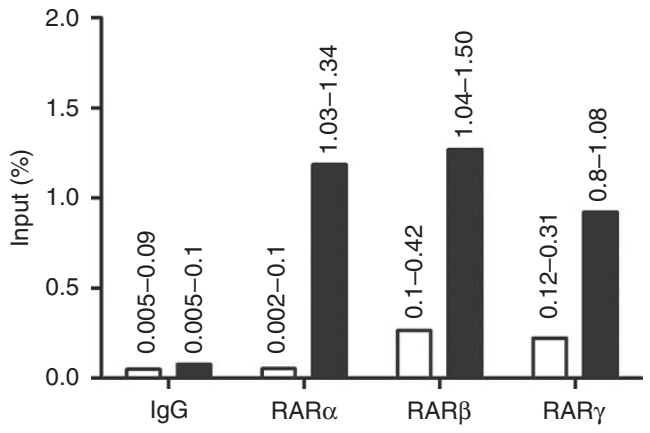

Figure 6 | Comparison of RA receptor binding to the Stra8 and RARb promoters in ovary. (a) The promoter region of mouse Stra8 is displayed showing the location of a putative RARE as well as binding sites for PCR primers used for chromatin immunoprecipitation (ChIP) analysis. (b) ChIP results for Stra8 using RAR antibodies compared to input DNA (diluted 100-fold) detected with primers flanking the Stra8 RARE. (c) ChIP results for $R A R b$ detected with primers flanking its RARE. (d) Quantitation of RAR binding (\% input) from the above ChIP results was performed by determining the amount of RAR-specific signal compared with input DNA for either Stra8 (open bar) or RARb (solid bar); ChIP results are from two independent experiments using different chromatin preparations (range is indicated above bars). IgG, antibody negative control; IP, immunoprecipitate; $M$, markers for DNA size in kilobase pairs (kb): NS, non-specific primers for region located several kb from either Stra8 or $R A R b$ RAREs; $S$, specific primers for RARE.

detection of RA activity in embryonic tissues ${ }^{19}$. Tissues from wild-type and Raldh2 ${ }^{-}$ embryos carrying $R A R E$-lac $Z$ were dissected free from the embryo, fixed, then stained with X-gal. RA activity in ovary and mesonephros was also examined using a tissue explant RA bioassay using the Sil-15 F9-RARE-lacZ RA-reporter cell line en, $^{20,3}$. In this bioassay, pieces of ovary and mesonephros were dissected cleanly away from each other and the portion of the ovary next to the mesonephros was discarded; tissues were cultured overnight on a monolayer of F9 cells carrying a RARE-lacZ transgene, followed by staining of the reporter cells with X-gal.

Gonad culture. Methods for culture and ketoconazole treatment of testis were similar to those previously reported ${ }^{3}$. Tissues were dissected from E13.5 embryos in PBS. Intact testes/mesonephros complexes and testes alone were cultured in Millicell culture plate inserts (Millipore) at $37^{\circ} \mathrm{C}$ in $5 \% \mathrm{CO}_{2}$. Tissues were cultured for $48 \mathrm{~h}$ in retinoid-free DMEM/F-12 culture media (Gibco-Life Technologies), containing either $0.7 \mu \mathrm{M}$ ketoconazole (Sigma Chemical) dissolved in $0.1 \%$ dimethyl sulfoxide or vehicle only (untreated). After $24 \mathrm{~h}$, culture medium was replaced with fresh medium supplemented with $0.7 \mu \mathrm{M}$ ketoconazole or vehicle. At the completion of the $48 \mathrm{~h}$ culture period, tissues were washed twice in PBS and fixed in $4 \%$ paraformaldehyde at $4{ }^{\circ} \mathrm{C}$ overnight, then processed for whole-mount in situ hybridization using a Stra8 riboprobe; alternatively, some testis/mesonephros samples were stained for RARE-lacZ activity with X-gal. RA treatment of E13.5 ovary/mesonephros was performed by culture in the above medium for $18 \mathrm{~h}$ in the absence or presence of all-trans-RA (Sigma Chemical) at 25 or $100 \mathrm{nM}$, followed by staining for RARE-lacZ activity with X-gal.
Chromatin immunoprecipitation. ChIP was performed according to the manufacturer's ChIP protocol (Active Motif). A total of 68 intact ovary/mesonephros complexes from E13.5 wild-type mouse embryos were dissected and crosslinked with $1 \%$ formaldehyde at room temperature for $15 \mathrm{~min}$. Isolated nuclei (in $650 \mu \mathrm{l}$ ) were sonicated for $20-23$ pulses of $10 \mathrm{~s}$ each on ice at $40 \%$ output amplitude using a 1.6-mm microtip probe from Misonix Digital Sonicator 4000 (Cole-Parmer Instrument Company) to shear DNA to an average size of $500 \mathrm{bp}$ followed by centrifugation at $15,700 \mathrm{~g}$ for $10 \mathrm{~min}$ at $4^{\circ} \mathrm{C}$. At this point, a small portion of supernatant was stored as input control. For each ChIP reaction, immunoprecipitation was performed using $150 \mu \mathrm{l}$ of sheared chromatin mixed with $3 \mu \mathrm{g}$ of either ChIP-grade anti-RAR- $\alpha$ (sc-551, Santa Cruz Biotechnology), anti-RAR- $\beta$ (Affinity Bioreagents), anti-RAR- $\gamma$ (sc-550 Santa Cruz Biotechnology), or control IgG (Cell Signaling) along with $25 \mu \mathrm{l}$ preblocked protein G-coated magnetic beads (Active Motifs) for $4 \mathrm{~h}$ at $4{ }^{\circ} \mathrm{C}$. Beads were washed thoroughly, and eluted DNA-protein complexes were reverse crosslinked and purified. The purified immunoprecipitated DNA was analysed by PCR, and was compared with input DNA that was diluted 100-fold; PCR products were examined by $3 \%$ agarose gel electrophoresis. ChIP analysis was performed in two independent experiments. Quantitation of RAR binding (\% input) was performed by determining the amount of RAR-specific signa compared with input DNA from two independent experiments. RARE-specific and non-specific primer sequences for mouse Stra 8 and $R A R b$ promoters used in this study were as follows:

Stra8 RARE-Fwd: 5'-ACTGCTACTGGGACCTTGAAGATG-3" Stra8 RARE-Rev: 5'-CTCTCAGAACACAAACGGTAGGAG-3' Stra8-NS-Fwd: 5'-TGACCACACAGATCTTGAACTTGC-3' Stra8-NS-Rev: $5^{\prime}$-CACGCCTGGTCTTCATGCACTA- ${ }^{\prime}$ $R A R b$ RARE-Fwd: $5^{\prime}$-TGGCATTGTTTGCACGCTGA-3' RARb RARE-Rev: $5^{\prime}$-CCCCCCTTTGGCAAAGAATAGA- ${ }^{\prime}$

$R A R b$ NS-F: $5^{\prime}$-AGTACAGACCTTCCAAGAGTGCCT- $3^{\prime}$ RARb NS-R: 5'-GTCATGGGAAAGAGAGGTTGAGC-3'

\section{References}

1. McLaren, A. Primordial germ cells in the mouse. Dev. Biol. 262, 1-15 (2003).

2. McLaren, A. \& Southee, D. Entry of mouse embryonic germ cells into meiosis. Dev. Biol. 187, 107-113 (1997).

3. Koubova, J. et al. Retinoic acid regulates sex-specific timing of meiotic initiation in mice. Proc. Natl Acad. Sci. USA 103, 2474-2479 (2006).

4. Bowles, J. et al. Retinoid signaling determines germ cell fate in mice. Science 312, 596-600 (2006)

5. Li, H., MacLean, G., Cameron, D., Clagett-Dame, M. \& Petkovich, M. Cyp26b1 expression in murine Sertoli cells is required to maintain male germ cells in an undifferentiated state during embryogenesis. PloS One 4, e7501 (2009).

6. MacLean, G., Li, H., Metzger, D., Chambon, P. \& Petkovich, M. Apoptotic extinction of germ cells in testes of Cyp26b1 knockout mice. Endocrinology 148, 4560-4567 (2007).

7. MacLean, G. et al. Cloning of a novel retinoic-acid metabolizing cytochrome P450, Cyp26B1, and comparative expression analysis with Cyp26A1 during early murine development. Mech. Dev. 107, 195-201 (2001).

8. Duester, G. Retinoic acid synthesis and signaling during early organogenesis. Cell 134, 921-931 (2008).

9. Anderson, E. L. et al. Stra8 and its inducer, retinoic acid, regulate meiotic initiation in both spermatogenesis and oogenesis in mice. Proc. Natl Acad. Sci. USA 105, 14976-14980 (2008).

10. Bowles, J. \& Koopman, P. Retinoic acid, meiosis and germ cell fate in mammals Development 134, 3401-3411 (2007).

11. Mic, F. A., Haselbeck, R. J., Cuenca, A. E. \& Duester, G. Novel retinoic acid generating activities in the neural tube and heart identified by conditional rescue of Raldh2 null mutant mice. Development 129, 2271-2282 (2002).

12. Niederreither, K., Subbarayan, V., Dollé, P. \& Chambon, P. Embryonic retinoic acid synthesis is essential for early mouse post-implantation development. Nat. Genet. 21, 444-448 (1999).

13. Mic, F. A., Molotkov, A., Benbrook, D. M. \& Duester, G. Retinoid activation of retinoic acid receptor but not retinoid $\mathrm{X}$ receptor is sufficient to rescue lethal defect in retinoic acid synthesis. Proc. Natl Acad. Sci. USA 100, 7135-7140 (2003).

14. Zhao, X. et al. Retinoic acid promotes limb induction through effects on body axis extension but is unnecessary for limb patterning. Curr. Biol. 19, 1050-1057 (2009).

15. Zhao, X., Brade, T., Cunningham, T. J. \& Duester, G. Retinoic acid controls expression of tissue remodeling genes Hmgn1 and Fgf18 at the digit-interdigit junction. Dev. Dyn. 239, 665-671 (2010).

16. Menke, D. B., Koubova, J. \& Page, D. C. Sexual differentiation of germ cells in XX mouse gonads occurs in an anterior-to-posterior wave. Dev. Biol. 262, 303-312 (2003).

17. Di Carlo, A. D., Travia, G. \& De Felici, M. The meiotic specific synaptonemal complex protein SCP3 is expressed by female and male primordial germ cells of the mouse embryo. Inter. J. Develop. Biol. 44, 241-244 (2000).

18. Rogakou, E. P., Pilch, D. R., Orr, A. H., Ivanova, V. S. \& Bonner, W. M. DNA double-stranded breaks induce histone H2AX phosphorylation on serine 139. J. Biol. Chem. 273, 5858-5868 (1998). 
19. Rossant, J., Zirngibl, R., Cado, D., Shago, M. \& Giguère, V. Expression of a retinoic acid response element- $h$ splac $Z$ transgene defines specific domains of transcriptional activity during mouse embryogenesis. Genes Dev. 5, 1333-1344 (1991).

20. Wagner, M., Han, B. \& Jessell, T. M. Regional differences in retinoid release from embryonic neural tissue detected by an in vitro reporter assay. Development 116, 55-66 (1992).

21. Horton, C. \& Maden, M. Endogenous distribution of retinoids during normal development and teratogenesis in the mouse embryo. Dev. Dyn. 202, 312-323 (1995).

22. Lin, Y., Gill, M. E., Koubova, J. \& Page, D. C. Germ cell-intrinsic and -extrinsic factors govern meiotic initiation in mouse embryos. Science 322, 1685-1687 (2008).

23. Oulad-Abdelghani, M. et al. Characterization of a premeiotic germ cell-specific cytoplasmic protein encoded by Stra8, a novel retinoic acid-responsive gene. J. Cell Biol. 135, 469-477 (1996).

24. Giuili, G. et al. Murine spermatogonial stem cells: targeted transgene expression and purification in an active state. EMBO Rep. 3, 753-759 (2002).

25. Mendelsohn, C., Ruberte, E., LeMeur, M., Morriss-Kay, G. \& Chambon, P. Developmental analysis of the retinoic acid-inducible RAR- $\beta 2$ promoter in transgenic animals. Development 113, 723-734 (1991).

26. Kurlandsky, S. B., Gamble, M. V., Ramakrishnan, R. \& Blaner, W. S. Plasma delivery of retinoic acid to tissues in the rat. J. Biol. Chem. 270, 17850-17857 (1995).

27. Schupp, M., Curtin, J. C., Kim, R. J., Billin, A. N. \& Lazar, M. A. A widely used retinoic acid receptor antagonist induces peroxisome proliferator-activated receptor-gamma activity. Mol. Pharmacol. 71, 1251-1257 (2007).

28. Sophos, N. A. \& Vasiliou, V. Aldehyde dehydrogenase gene superfamily: the 2002 update. Chem. Biol. Interact 143, 5-22 (2003).

29. Best, D., Sahlender, D. A., Walther, N., Peden, A. A. \& Adams, I. R. Sdmg1 is a conserved transmembrane protein associated with germ cell sex determination and germline-soma interactions in mice. Development 135, 1415-1425 (2008).

30. Trautmann, E. et al. Retinoic acid prevents germ cell mitotic arrest in mouse fetal testes. Cell Cycle 7, 656-664 (2008).

31. Guerquin, M. J. et al. New testicular mechanisms involved in the prevention of fetal meiotic initiation in mice. Dev. Biol. 346, 320-330 (2010).
32. Bowles, J. et al. FGF9 suppresses meiosis and promotes male germ cell fate in mice. Dev. Cell 19, 440-449 (2010).

33. Molotkov, A., Molotkova, N. \& Duester, G. Retinoic acid guides eye morphogenetic movements via paracrine signaling but is unnecessary for retinal dorsoventral patterning. Development 133, 1901-1910 (2006).

34. Wilkinson, D. G. \& Nieto, M. A. Detection of messenger RNA by in situ hybridization to tissue sections and whole mounts. Methods Enzymol. 225, 361-373 (1993).

35. Molotkova, N., Molotkov, A. \& Duester, G. Role of retinoic acid during forebrain development begins late when Raldh3 generates retinoic acid in the ventral subventricular zone. Dev. Biol. 303, 601-610 (2007).

\section{Acknowledgments}

We thank D. Page for mouse Stra 8 cDNA and J. Bowles for mouse Scp3 cDNA used to prepare in situ hybridization probes, M. Weitzman for $\gamma$-H2AX antibody, J. Rossant for providing RARE-lacZ mice and M. Wagner for the Sil-15 F9-RARE-lacZ RA-reporter cell line. This work was funded by National Institutes of Health Grant GM062848 (G.D.).

\section{Author contributions}

S.K. and G.D. designed the study, analysed the data and wrote the paper. S.K. performed all the experiments, C.C. assisted with the F9 reporter cell assay. T.B., T.C. and X.Z. provided valuable reagents for the study. All authors discussed the results and commented on the manuscript.

\section{Additional information}

Competing financial interests: The authors declare no competing financial interests.

Reprints and permission information is available online at http://npg.nature.com/ reprintsandpermissions/

How to cite this article: Kumar, S. et al. Sex-specific timing of meiotic initiation is regulated by Cyp26b1 independent of retinoic acid signalling. Nat. Commun. 2:151 doi: $10.1038 /$ ncomms1136 (2011) 